\title{
¿CUÁNTO CONTRIBUYE LA ECONOMÍA AZUL A UNA REGIÓN? UNA APROXIMACIÓN METODOLÓGICA APLICADA A LA ECONOMÍA DE CATALUÑA
}

\author{
Martin Alba*, Lourdes Reig, David Fernández, Xavier Díaz
}

CREDA UPC IRTA (Castelldefels, martin.federico@upc.edu)

Palabras clave: Economía azul, Dimensión económica, Cataluña

\section{Resumen}

Este trabajo tiene por objetivo realizar una aproximación metodológica al dimensionamiento de la economía azul (concepto que integra los sectores económicos relacionados con el mar y con el medio costero) en el PIB y en la ocupación de una región. Para ello se ha utilizado como base la metodología implementada en el "EU Blue Economy Report 2019", publicado por la Comisión Europea, pero desarrollando las adaptaciones necesarias a un contexto regional. El análisis se ha focalizado en la región de Cataluña para el año 2017. Combinando diferentes fuentes estadísticas y métodos de ponderación, el análisis ha permitido dimensionar la aportación económica de los sectores ligados al mar y al medio costero en un 3,4\% del PIB (7.295,4 millones de euros) y en un 5,9\% de la ocupación total (215.364 personas). Aun teniendo en cuenta las especificidades de la economía catalana, este trabajo puede servir de referencia para otros esfuerzos regionales de medición de la economía azul.

\section{Introducción y objetivos}

El objetivo de este estudio ha sido realizar una aproximación metodológica para estimar el peso económico de los sectores vinculados a la economía azul en Cataluña.

El trabajo utiliza como principal referencia metodológica el informe anual "EU Blue Economy Report 2019" publicado por la Comisión Europea (2020), el cual aporta los fundamentos metodológicos de estimación a nivel nacional. Nuestro trabajo, utiliza esos lineamientos, pero aporta elementos diferenciales para poder aplicar la metodología a nivel regional (Cataluña).

Por economía azul, hacemos referencia a todos los sectores/actividades económicas vinculadas al mar y a la costa. Estos sectores, son identificados en informe comentado según se muestra en la tabla 1. En ella se muestra que forman parte de la economía azul 6 grandes sectores y 11 subsectores (que integran a su vez 48 actividades económicas detalladas a 4 dígitos del CNAE 2009).

Tabla 1. Actividades y sectores económicos que conforman la economía azul

\begin{tabular}{|c|c|c|c|}
\hline \multirow{2}{*}{ Sector } & \multirow{2}{*}{ Subsectores } & \multicolumn{2}{|c|}{ Actividades económicas (CNAE 2009) } \\
\hline & & Código & Descripción \\
\hline \multirow{9}{*}{$\begin{array}{l}\text { 1. Recurs } \\
\text { os marinos vivos }\end{array}$} & \multirow{2}{*}{$\begin{array}{l}1.1 . \quad \text { Pesca y } \\
\text { acuicultura marina }\end{array}$} & 0311 & Pesca marina \\
\hline & & 0321 & Acuicultura marina \\
\hline & \multirow{7}{*}{$\begin{array}{l}\text { 1.2. Procesamiento y } \\
\text { distribución }\end{array}$} & 1021 & Procesado de pescados, crustáceos y moluscos \\
\hline & & 1022 & Fabricación de conservas de pescado \\
\hline & & 1040 & Fabricación de aceites y grasas vegetales y animales \\
\hline & & 1085 & Elaboración de platos y comidas preparados \\
\hline & & 1089 & Elaboración de otros productos alimenticios n.c.o.p. \\
\hline & & 4638 & $\begin{array}{l}\text { Comercio al por mayor de pescados y mariscos y otros productos } \\
\text { alimenticios }\end{array}$ \\
\hline & & 4723 & $\begin{array}{l}\text { Comercio al por menor de pescados y mariscos en establecimientos } \\
\text { especializados }\end{array}$ \\
\hline \multirow{5}{*}{$\begin{array}{l}2 . \quad \text { Otros } \\
\text { recursos marinos }\end{array}$} & \multirow{5}{*}{$\begin{array}{l}\text { 2.1. Petróleo, gas y } \\
\text { otros minerales* }\end{array}$} & 0610 & Extracción de crudo de petróleo \\
\hline & & 0620 & Extracción de gas natural \\
\hline & & 0910 & Actividades de apoyo a la extracción de petróleo y gas natural \\
\hline & & 0812 & Extracción de gravas y arenas; extracción de arcilla y caolín \\
\hline & & 0990 & Actividades de apoyo a otras industrias extractivas \\
\hline \multirow{4}{*}{$\begin{array}{l}3 . \quad \text { Activi } \\
\text { dades portuarias }\end{array}$} & \multirow{3}{*}{$\begin{array}{l}\text { 3.1. Puertos y } \\
\text { almacenaje }\end{array}$} & 5222 & $\begin{array}{l}\text { Actividades anexas al transporte marítimo y por vías navegables } \\
\text { interiores }\end{array}$ \\
\hline & & 5224 & Manipulación de mercancías \\
\hline & & 5210 & Depósito y almacenamiento \\
\hline & $\begin{array}{l}\text { 3.2. Construcción de } \\
\text { obras hidráulicas }\end{array}$ & 4291 & Obras hidráulicas \\
\hline \multirow{3}{*}{$\begin{array}{l}\text { 4. Constr } \\
\text { ucción naval }\end{array}$} & \multirow{3}{*}{$\begin{array}{l}4.1 . \\
\text { naval }\end{array}$} & 3011 & Construcción de barcos y estructuras flotantes \\
\hline & & 3012 & Construcción de embarcaciones de recreo y deporte \\
\hline & & 3315 & Reparación y mantenimiento naval \\
\hline
\end{tabular}




\begin{tabular}{|c|c|c|c|}
\hline \multirow{7}{*}{ Sector } & \multirow{2}{*}{ Subsectores } & \multicolumn{2}{|c|}{ Actividades económicas (CNAE 2009) } \\
\hline & & Código & Descripción \\
\hline & \multirow{5}{*}{$\begin{array}{l}\text { 4.2. Equipo y } \\
\text { maquinaria naval }\end{array}$} & 1392 & $\begin{array}{l}\text { Fabricación de artículos confeccionados con textiles, excepto } \\
\text { prendas de vestir }\end{array}$ \\
\hline & & 1394 & Fabricación de cuerdas, cordeles, bramantes y redes \\
\hline & & 2651 & $\begin{array}{l}\text { Fabricación de instrumentos y aparatos de medida, verificación y } \\
\text { navegación }\end{array}$ \\
\hline & & 2811 & $\begin{array}{l}\text { Fabricación de motores y turbinas, excepto los destinados a } \\
\text { aeronaves, vehículos automóviles y ciclomotores }\end{array}$ \\
\hline & & 3230 & Fabricación de artículos de deporte \\
\hline \multirow{6}{*}{$\begin{array}{l}5 . \quad \text { Transp } \\
\text { orte Marítimo }\end{array}$} & \multirow{6}{*}{$\begin{array}{l}\text { 5.1. Expedición y } \\
\text { transporte }\end{array}$} & 5010 & Transporte marítimo de pasajeros \\
\hline & & 5020 & Transporte marítimo de mercancías \\
\hline & & 5030 & Transporte de pasajeros por vías navegables interiores \\
\hline & & 5040 & Transporte de mercancías por vías navegables interiores \\
\hline & & 5229 & Otras actividades anexas al transporte \\
\hline & & 7734 & Alquiler de medios de navegación \\
\hline \multirow{16}{*}{$\begin{array}{l}6 . \quad \text { Turism } \\
\text { o de costa }\end{array}$} & \multirow{4}{*}{ Alojamiento } & 5510 & Hoteles y alojamientos similares \\
\hline & & 5520 & Alojamientos turísticos y otros alojamientos de corta estancia \\
\hline & & 5530 & Campings y aparcamientos para caravanas \\
\hline & & 5590 & Otros alojamientos \\
\hline & \multirow{6}{*}{ 6.2. Transporte } & 4730 & $\begin{array}{l}\text { Comercio al por menor de combustible para la automoción en } \\
\text { establecimientos especializados }\end{array}$ \\
\hline & & 4910 & Transporte interurbano de pasajeros por ferrocarril \\
\hline & & 4931 & Transporte terrestre urbano y suburbano de pasajeros \\
\hline & & 4932 & Transporte por taxi \\
\hline & & 4939 & Tipos de transporte terrestre de pasajeros n.c.o.p. \\
\hline & & 5110 & Transporte aéreo de pasajeros \\
\hline & \multirow{6}{*}{ 6.3. Otros gastos } & 4760 & $\begin{array}{l}\text { Comercio al por menor de artículos culturales y recreativos en } \\
\text { establecimientos especializados }\end{array}$ \\
\hline & & 4770 & $\begin{array}{l}\text { Comercio al por menor de otros artículos en establecimientos } \\
\text { especializados }\end{array}$ \\
\hline & & 5610 & Restaurantes y puestos de comidas \\
\hline & & 5621 & Provisión de comidas preparadas para eventos \\
\hline & & 5629 & Otros servicios de comidas \\
\hline & & 5630 & Establecimientos de bebidas \\
\hline
\end{tabular}

*Estas actividades no se han podido incluir en el informe por falta de datos. Fuente: elaboración propia en base a Comisión Europea (2020)

\section{Metodología}

Para aproximar la dimensión económica de estos sectores y actividades económicas se ha trabajado con tres macro magnitudes principales: Volumen de negocios; Valor añadido y Empleo. Los datos se obtienen de varias fuentes según sea el sector analizado. Las fuentes principales son:

- Encuestas estructurales de empresas: elaboradas por el INE y disponibles bajo solicitud a nivel regional en el IDESCAT.

- Estadística industrial de productos (INE)

- Encuestas económicas de la Pesca y de la Acuicultura: elaboradas por el Ministerio de agricultura, y disponibles a nivel regional bajo solicitud especifica

- Encuestas de gasto del turismo: disponible en el INE.

- Estadística de ocupación hotelera del INE.

Ahora bien, muchos de las actividades económicas (clasificadas a 4 dígitos del CNAE) incluidas en la economía azul no destinan el $100 \%$ de su producción a actividades marítimas. En estos sectores industriales manufactureros, la ponderación se realiza con la Estadística industrial de productos (INE-IDESCAT). El detalle de esta estadística permite obtener el peso porcentual de la producción de productos objetivo sobre el total de la actividad en cuestión. Este procedimiento se ha realizado en todas las actividades industriales que ha sido posible según el detalle de la tabla 2.

Tabla 2. Ponderaciones realizadas en actividades económicas no $100 \%$ marítimas

\begin{tabular}{|c|c|c|}
\hline \multicolumn{2}{|c|}{ CNAE-2009 } & \multirow{3}{*}{$\begin{array}{l}\text { Proporción marítima de la actividad } \\
\text { Porcentaje de productos de la industria pesquera sobre } \\
\text { el total de producción de estas } 3 \text { actividades. Fuente } \\
\text { encuesta industrial de productos (códigos } 10411200 \text {, } \\
10851200,10851410,10891400 \text { ) }\end{array}$} \\
\hline 1040 & $\begin{array}{l}\text { Fabricación de aceites y grasas vegetales } \\
\text { y animales }\end{array}$ & \\
\hline 1085 & $\begin{array}{l}\text { Elaboración de platos y comidas } \\
\text { preparados }\end{array}$ & \\
\hline
\end{tabular}




\begin{tabular}{|c|c|c|}
\hline \multicolumn{2}{|c|}{ CNAE-2009 } & \multirow[t]{2}{*}{ Proporción marítima de la actividad } \\
\hline 1089 & $\begin{array}{l}\text { Elaboración de otros productos } \\
\text { alimenticios n.c.o.p. }\end{array}$ & \\
\hline 5224 & Manipulación de mercancías & \multirow{2}{*}{$\begin{array}{l}50 \% \text { (Porcentaje utilizado en el Blue Economy Report } \\
\text { por falta de información) }\end{array}$} \\
\hline 5210 & Depósito y almacenamiento & \\
\hline 1392 & $\begin{array}{l}\text { Fabricación de artículos confeccionados } \\
\text { con textiles, excepto prendas de vestir }\end{array}$ & \multirow{5}{*}{$\begin{array}{l}\text { Porcentaje de productos vinculados a la industria naval } \\
\text { y pesquera sobre el total de producción de estas } 6 \\
\text { actividades. Fuente encuesta industrial (códigos } \\
13922250,13922999,13941233,13941235,26511180 \text {, } \\
26512080,28111100,28111200,28111311, \\
28111315,28111319,28112200,32301300,32301600 \text { ) }\end{array}$} \\
\hline 1394 & $\begin{array}{l}\text { Fabricación de cuerdas, cordeles, } \\
\text { bramantes y redes }\end{array}$ & \\
\hline 2651 & $\begin{array}{l}\text { Fabricación de instrumentos y aparatos de } \\
\text { medida, verificación y navegación }\end{array}$ & \\
\hline 2811 & $\begin{array}{l}\text { Fabricación de motores y turbinas, } \\
\text { excepto los destinados a aeronaves, } \\
\text { vehículos automóviles y ciclomotores }\end{array}$ & \\
\hline 3230 & Fabricación de artículos de deporte & \\
\hline 5229 & Otras actividades anexas al transporte & $\begin{array}{l}50 \% \text { (Porcentaje utilizado en el Blue Economy Report } \\
\text { por falta de información) }\end{array}$ \\
\hline
\end{tabular}

Fuente: elaboración propia en base a Comisión Europea (2020)

Para el caso del turismo de costa o marítimo, el trabajo de la Comisión Europea (2020), también desarrolla un método específico. Para estimar el valor de negocios, valor añadido y empleo de este sector, partimos de los datos de gasto del turismo en las partidas: Alojamiento, Transporte y Otros gastos, disponibles en la encuesta de gasto del turismo (INE). Una vez obtenidos esos datos se calcula el porcentaje que representa ese gasto por partidas, en el volumen de negocios de las actividades económicas correspondientes disponibles en las encuestas estructurales de empresas. Ese porcentaje se utiliza también para obtener el valor añadido y el volumen de empleo.

Este procedimiento permite conocer el peso del turismo (total), pero para aproximar la proporción costera/marítima se realiza otra ponderación, esta vez utilizando el peso de las pernoctaciones en zonas costeras sobre el total de la Comunidad Autónoma (disponible en la Encuesta de ocupación hotelera del INE). En el caso de Cataluña es del 57,3\% .

\section{Resultados}

- Los sectores de la economía azul en Cataluña generan conjuntamente un valor de negocios 25.032 millones de euros, lo cual representa un 5,8\% de la producción total de Cataluña. Por otra parte, representa un 31,3\% de la economía azul en España.

- Estos sectores a su vez, generan un valor añadido bruto (VAB) de 7.295,4 millones de euros, que representan el 3,4\% del valor añadido bruto total generado en Cataluña. Además, representa un 27,7\% de la economía azul en España.

- Finalmente, los sectores de la economía azul generan un total de 215.364 empleos directos, lo cual representa un 5,9\% del total de personas ocupadas en Cataluña. A su vez, representa un $28,3 \%$ de la economía azul en España.

- Los principales subsectores económicos más importantes por su dimensión son

- El turismo marítimo con un $68 \%$ del VAB y un $79 \%$ del empleo.

- El transporte marítimo con un $12 \%$ del VAB y un $9 \%$ del empleo.

- Los recursos marinos vivos con un 9\% del VAB i un 7\% del empleo.

- Las actividades portuarias, con un $9 \%$ del VAB y un $4 \%$ del empleo.

Tabla 3. Principales Macromagnitudes de los sectores que forman parte de la Economía azul en Cataluña

\begin{tabular}{|c|c|c|c|c|c|c|c|}
\hline \multirow{2}{*}{ Sector } & \multirow{2}{*}{ Subsector } & \multicolumn{2}{|c|}{ VAB } & \multicolumn{2}{|c|}{ Empleo } & \multicolumn{2}{|c|}{$\begin{array}{l}\text { Volumen de } \\
\text { negocios }\end{array}$} \\
\hline & & M€ & $\%$ & M€ & $\%$ & $\mathbf{M} €$ & $\%$ \\
\hline \multirow{2}{*}{$\begin{array}{l}\text { 1. Recursos } \\
\text { marinos vivos }\end{array}$} & 1.1. Pesca y acuicultura marina & 74,3 & $1,0 \%$ & 3.614 & $1,7 \%$ & 145,2 & $0,6 \%$ \\
\hline & $\begin{array}{l}\text { 1.2. Procesamiento y } \\
\text { distribución }\end{array}$ & 566,7 & $7,8 \%$ & 12.742 & $5,9 \%$ & $4.512,4$ & $18,0 \%$ \\
\hline $\begin{array}{l}\text { 2. Otros recursos } \\
\text { marinos }\end{array}$ & $\begin{array}{l}\text { 2.1. Petróleo, gas y otros } \\
\text { minerales }\end{array}$ & \multicolumn{6}{|c|}{ No disponible } \\
\hline & 3.1. Puertos y almacenaje & 575,4 & $7,9 \%$ & 6.778 & $3,1 \%$ & $1.215,7$ & $4,9 \%$ \\
\hline
\end{tabular}

${ }^{5}$ Sin considerar la ciudad de Barcelona, dado que incluyendo todas las pernoctaciones en la ciudad este porcentaje superaría el 75\% del total. 


\begin{tabular}{|c|c|c|c|c|c|c|c|}
\hline \multirow{3}{*}{\begin{tabular}{l}
\multicolumn{1}{c}{ Sector } \\
3. Actividades \\
portuarias
\end{tabular}} & \multirow{2}{*}{ Subsector } & \multicolumn{2}{|c|}{ VAB } & \multicolumn{2}{|c|}{ Empleo } & \multicolumn{2}{|c|}{$\begin{array}{c}\text { Volumen de } \\
\text { negocios }\end{array}$} \\
\hline & & $\mathbf{M €}$ & $\%$ & M€ & $\%$ & $\mathbf{M} €$ & $\%$ \\
\hline & $\begin{array}{l}\text { 3.2. Construcción de obras } \\
\text { hidráulicas }\end{array}$ & 76,2 & $1,0 \%$ & 1.320 & $0,6 \%$ & 242,6 & $1,0 \%$ \\
\hline \multirow{2}{*}{$\begin{array}{l}\text { 4. Construcción } \\
\text { naval }\end{array}$} & 4.1. Construcción naval & 69,2 & $0,9 \%$ & 1.406 & $0,7 \%$ & 207,8 & $0,8 \%$ \\
\hline & 4.2. Equipo y maquinaria naval & 40,2 & $0,6 \%$ & 846 & $0,4 \%$ & 137,8 & $0,6 \%$ \\
\hline $\begin{array}{l}\text { 5. Transporte } \\
\text { Marítimo }\end{array}$ & 5.1. Expedición y transporte & 906,4 & $12,4 \%$ & 19.612 & $9,1 \%$ & $4.527,9$ & $18,1 \%$ \\
\hline \multirow{3}{*}{$\begin{array}{l}\text { 6. Turismo de } \\
\text { costa }\end{array}$} & 6.1. Alojamiento & $1.240,7$ & $17,0 \%$ & 27.876 & $12,9 \%$ & $2.590,6$ & $10,3 \%$ \\
\hline & 6.2. Transporte & $1.263,1$ & $17,3 \%$ & 26.241 & $12,2 \%$ & $3.641,9$ & $14,5 \%$ \\
\hline & 6.3. Otros gastos & $2.483,2$ & $34,0 \%$ & 114.929 & $53,4 \%$ & $7.810,1$ & $31,2 \%$ \\
\hline \multirow{3}{*}{ Total } & Total Economía Azul Cataluña & $7.295,4$ & $100,0 \%$ & 215.364 & $100,0 \%$ & $25.032,0$ & $100,0 \%$ \\
\hline & $\%$ en la Economía de Catalunya & $3,4 \%$ & & $5,9 \%$ & & $5,8 \%$ & \\
\hline & $\begin{array}{l}\text { \% en la Economía Azul en } \\
\text { España }\end{array}$ & $27,7 \%$ & & $28,3 \%$ & & $31,3 \%$ & \\
\hline
\end{tabular}

Fuente: elaboración propia en base Comisión Europea (2020) y a datos de IDESCAT, INE, MAPA.

\section{Conclusiones}

El presente trabajo ha tenido por objetivo realizar una aproximación metodológica para dimensionar la economía azul a nivel regional. La base metodológica es el informe publicado anualmente por la Comisión Europea que dimensiona las principales Macromagnitudes, pero a nivel de Estados Miembro. Nuestra aproximación aporta como elemento diferencial el enfoque regional.

La aproximación se realizó en Cataluña y se ha demostrado la aplicabilidad de la misma, siempre y cuando se disponga en otras regiones de información suficiente en cuatro fuentes de datos principales del INE: La encuesta estructural de empresas, la encuesta industrial de productos, las encuestas de gasto del turismo y finalmente, la encuesta de ocupación hotelera por zonas costeras.

En cuanto a los resultados obtenidos, se demuestra la importancia de los sectores económicos vinculados al mar en Cataluña, con una aportación del 3,4\% del PIB; 5,9\% de la ocupación y del 5,8\% del volumen de negocios. En esta aportación es el turismo de costa el que más aporta, con un $68 \%$ del valor añadido y un $79 \%$ del empleo del total de la economía azul catalana.

\section{Bibliografía}

- European Commission (2020). The EU Blue Economy Report 2019. Oficina de publicaciones de la Unión Europea. Luxemburgo.

- Instituto Nacional de Estadística (INE) - IDESCAT (2018)

- Estadística estructural de empresas del sector industrial (2017)

- Estadística estructural de empresas del sector comercio (2017)

- Estadística estructural de empresas del sector servicios (2017)

- Estadística industrial de productos (2017)

- Estadística de gasto del turismo extranjero; de residentes de Catalunya y de los residentes del resto de España (2017)

- Instituto Nacional de Estadística (INE)

- Encuesta de ocupación hotelera (2017)

- Ministerio de Agricultura, Pesca y Alimentación.

- Encuesta económica de la pesca marítima (2017)

- Encuesta económica de la acuicultura (2017)

\section{Agradecimientos}

Los autores agradecen el apoyo brindado por la Dirección General de Pesca y Asuntos Marítimos del Departamento de Acción Climática, Alimentación y Agenda Rural de Cataluña de la Generalitat de Cataluña para la elaboración de este trabajo. 